\title{
El Delirio Erotomaníaco en el film de Almodovar "Hable con ella"
}

\author{
Prado Ordóñez Fernándeza
}

a FEA de la Unidad de Salud Mental del Hospital General de Ciudad Real.

\section{Dirección para}

correspondencia: María del Prado Ordoñez Fernandez. $\mathrm{C} /$ Alarcos $\mathrm{N}^{\circ} 3,1^{\circ} \mathrm{D}$,

13001-Ciudad Real. E-mail: mpordonez@sescam.jccm.es

Recibido el 17 de febrero de 2009.

Aceptado para su publicación el 28 de febrero de 2009.

\section{RESUMEN}

El delirio erotomaníaco es un subtipo de trastorno delirante en el que el tema central es la idea delirante de que una persona está enamorada del sujeto. La idea delirante suele referirse a un amor romántico idealizado, a una unión espiritual más que a una mera atracción sexual. En el film "Hable con ella", Almodóvar presenta la dinámica de este trastorno, que utiliza los mismos elementos de la razón y la pasión humana, para finalizar en un delirio de amor tan erróneo como imposible.

Palabras clave. Trastornos Mentales. Delirio.

\section{ABSTRACT}

Erotomanic delusion in Almodovar's film "Hable con ella"

Erotomania is a delusional disorder subtype in which the core symptom is that the subject is under the delusion that another person is in love with him or her. The delusion is usually of an idealised romantic love, a spiritual union rather than a mere sexual attraction. In his film "Hable con ella" Almodóvar presents the dynamics of this disorder, which uses the same elements of reasoning and human passion and ends in the delusion of an impossible love.

Key words. Mental Disorders. Delirium.

"Amar es dar lo que uno no tiene a alguien que no lo quiere" LACAN

\section{INTRODUCCIÓN}

\section{¿Qué es un delirio?}

Delirio es una falsa creencia basada en una inferencia incorrecta acerca de la realidad externa, que se sostiene con firmeza a pesar de la opinión de los demás y de las pruebas incontrovertibles y obvias que indican lo contrario.

\section{¿Qué es el delirio erotomaniaco?}

Según el Manual Diagnóstico y Estadístico de los Trastornos Mentales (DSM-IV-TR), el delirio erotomaníaco es un subtipo de trastorno delirante en el que el tema central es la idea delirante de que una persona está enamorada del sujeto. La idea delirante suele referirse a un amor romántico idealizado, a una unión espiritual más que a una mera atracción sexual.

En la literatura psiquiátrica, desde la antigüedad, los delirios erotomaníacos estaban protagonizados básicamente por mujeres, especialmente solitarias y frustradas solteronas, pero cada vez han sido informados más casos de erotomanía masculina (Taylor, 1983; Goldstein, 1987). En ambos sexos, la mayoría de los casos descritos implican emociones heterosexuales, pero la erotomanía homosexual está actualmente bien documentada en uno y otro sexo ${ }^{1}$.

\section{Evolución histórica del concepto}

Según la interesante Tesis de Ferdière, desde su inicio (Hipócrates de Cos, Galeno y Plutarco) hasta la primera treintena del siglo XVIII, la erotomanía estuvo confundida con la ninfomanía, que resulta de la exaltación desenfrenada del instinto sexual en la mujer. Su equivalente en el hombre lo constituía la satiriasis². 
Para Esquirol (1782-1840), la erotomanía difería esencialmente de la ninfomanía y de la satiriasis, ya que en la erotomanía el amor "estaba en la cabeza" ("l'amour est dans la tête"), considerándola como una monomanía intelectual, es decir, una forma de alineación mental que afecta parcialmente a la mente dejando las facultades intelectuales intactas, cuya causa es una pasión patológica que actúa sobre la inteligencia fijando su atención. La locura de amor, dice Esquirol, no respeta ni a sabios ni a locos, el enfermo es un juguete de la imaginación. El desorden intelectual, escribe, está concentrado sobre un solo objeto. Los enfermos parten de un principio falso, cuyos razonamientos lógicos siguen sin desviación ninguna, y del que obtienen consecuencias legítimas que modifican sus afectos y los actos de su voluntad. Ilusiones, alucinaciones, asociaciones erróneas de ideas, convicciones falsas, equivocadas y extrañas son la base de tal delirio. La denominó locura del amor casto ("folie de l'amour chaste") $)^{3}$.

Morel, en 1853, define ésta monomanía como "délire d'amour", imprimiéndole también un sesgo de amor platónico, sesgo reafirmado por Ball.

Magnan, en 1893, por primera vez conjetura que la erotomanía obedece a un trastorno del lóbulo frontal del cerebro.

Carl Wernicke (1848-1896), en sus célebres Lecciones de Psiquiatría adscribe la hipótesis de la "sejución": escisión de la fluidez del pensamiento, con la aparición de ideas autóctonas, prevalentes y sobrevaloradas, las cuales, mediante su emergencia, dan lugar a los delirios paranoicos, a veces con tonalidad erotomaníaca.

Sérieux (1864-1947) y Capgrás (1873-1950), en su Tratado sobre las Locuras Razonantes, vinculan la erotomanía con los delirios interpretativos, denominándola delirio erótico. La interpretación delirante es para los autores un razonamiento falso que tiene como punto de partida una sensación real, un hecho auténtico, el cual, en virtud de asociaciones de ideas ligadas a las tendencias, a la afectividad, y con la ayuda de inducciones o deducciones erróneas, adquiere una significación personal para el enfermo.

En la citada obra los autores definen este delirio como un delirio lúcido, generalmente con ausencia de alucinaciones, con conservación de la actividad psíquica, con evolución extensiva de las interpretaciones delirantes, incurable pero sin evolución demencial ${ }^{4}$.

Emil Kraepelin (1865-1926), en 1921, incluye los delirios erotomaníacos en los delirios paranoicos o paranoia ${ }^{5}$.

Gaétan Gatian de Clérambault (1872-1934), médico jefe de la enfermería especial de la Prefectura de Policía (institución a la que llegan toda clase de margi- nales traídos por la Policía), en la que ejerció durante treinta años, describe la erotomanía como la ilusión delirante de ser amado, trazando un cuadro clínico minucioso con tres fases: esperanza, despecho y rencor. Para de Clérambault, el comienzo es siempre brusco, preciso, a modo de "flechazo". En la sesión clínica de la Societé Clínique en la que presenta el "caso Leontine" comenta: "la pasión fue espontánea, las interpretaciones vinieron después", "nuestra enferma muestra de qué manera ha estado capturada, desde el primer día, por la mirada fascinante de su objeto", "este delirio pasional se establece sobre un nudo ideo-afectivo inicial, cuyo elemento afectivo está constituido por una emoción vehemente, profunda, destinada a perpetuarse y que acapara desde el primer día todas las fuerzas del espíritu con la ilusión de ser amado por el objeto".

La fábula delirante se constituía con cierta lógica a partir de elementos falsos (errores o ilusiones) que él llamó postulados ("me ama", "no hay dudas", "soy feliz", "mi vida tiene un nuevo sentido", "no puede ser feliz sin mí", "es libre"). Los componentes del sentimiento generador del postulado son orgullo, deseo y esperanza. Estos síndromes pasionales mórbidos, según el autor, se presentaban en ocasiones en formas autónomas o puras (erotomanía esencial) y otras veces asociadas a otros delirios intelectuales o alucinatorios (erotomanía mixta o asociada). El autor era de tendencia mecanicista y se opuso a toda interpretación psicológica de la psicosis y, aun reconociendo su genio observador, la calidad y la exactitud de sus descripciones, no podemos olvidar que sus informes eran peritajes judiciales tomados como sagrados por el juez de turno por su maestría y renombre, y sus entrevistas inquisidoras eran auténticos interrogatorios policiales $^{6}$.

H. Ey (1900-1977) introducía la erotomanía dentro de los delirios pasionales, caracterizados, en primer lugar, por la exaltación, en segundo lugar, por la idea prevalente que subordina todos los fenómenos psíquicos y todas las conductas a un postulado fundamental, el de una convicción inconmovible y, en tercer lugar, por su desarrollo en sector, en el sentido de que el delirio constituye un sistema parcial que penetra como una cuña en la realidad. Los estados pasionales delirantes, refiere el autor, tienen características clínicas que conviene subrayar: estos estados pasionales se producen sobre un fondo de desequilibrio caracterológico, se acompañan de un cortejo de trastornos (trastornos tímicos, experiencias alucinatorias, despersonalización, fases de exacerbación, impulsividad), dando lugar a períodos productivos que testifican un desquiciamiento de la vida psíquica, y son patológicos y delirantes porque la pasión, incluso si se encuentra inserta en una situación real, tiene una estructura esencialmente imaginaria. En efecto, o bien los acontecimientos y los personajes que lo 
componen son irreales, y éste es el caso en que el delirio es más evidente, o bien la realidad de la situación es ampliamente desbordada o eclipsada por la proyección de fantasmas más o menos inconscientes. Los delirios pasionales proceden en su misma estructura de la rigidez sistemática de la pasión que constituye el eje. Son bloques ideo-afectivos inconmovibles, impermeables a la experiencia y rebeldes a toda evidencia.

Aunque H. Ey acepta de G. de Clérambault todos los aspectos clínicos y evolutivos, discrepa sobre la ausencia de alucinaciones en este delirio, considerando que las "conversaciones indirectas" con el objeto son a menudo alucinatorias, estando comprendidas dentro de un vasto síndrome de influencia y de automatismo mental (presencia continua del objeto, influencia física del deseo del objeto, cohabitación y posesión erótica, declaraciones por transmisiones de pensamiento, etc. $)^{7}$.

En la escuela psicoanalista, Freud consideraba que la base del delirio era la proyección de contenidos no admitidos por el super-yo, proyección que desplaza deseos, tendencias o pulsiones sobre las personas o las cosas del mundo exterior, evitando, de esta manera, el displacer y la angustia. Al igual que en el sueño, en el delirio existe también un cumplimiento de deseos. Adler sentenciaba que el delirio servía para ocultar la derrota en la vida. Para Lacán, el delirio es secundario al surgimiento de un significante aislado, desatado en lo real. Este aislamiento patológico del significante es la marca de una alteración del tejido simbólico en el cual se encuadra el sujeto. La causa de esta alteración se debe al mecanismo de la forclusión del significante del nombre del padre. Si el neurótico habita el lenguaje y el significante, el psicótico es habitado por él.

En la escuela cognitivo-conductual, tradicionalmente se ha considerado que los delirios suponen una forma de pensamiento cualitativamente diferente a las creencias normales, insistiéndose también en la idea de que estos pensamientos eran insensibles a cualquier evidencia o explicación alternativa y, por tanto, inmodificables. Diferentes trabajos han demostrado que la convicción del pensamiento delirante no siempre es total e invariable y que hay veces que los delirios son sensibles a otras explicaciones, y por tanto pueden ser modificados (Alford, 1986; Garety, 1991 y 1992; Loewe y Chadwik, 1990).

Para explicar el mecanismo del pensamiento delirante se ha aludido a la presencia de sesgos atribucionales que facilitarían la aparición de inferencias erróneas.

En esta misma línea, otros autores (Garety, 1991) señalan sesgos en el razonamiento probabilístico de estos pacientes, lo que se apoya en estudios experimentales que demuestran que los pacientes con ideas delirantes tienden a emitir juicios y a sacar conclusiones sobre la base de menos información que las personas normales o que otros pacientes psiquiátricos.

Esta nueva perspectiva aportada por los enfoques cognitivos tiene importantes implicaciones de cara al tratamiento de los delirios, proporcionando un nuevo objeto y enfoque. El procedimiento propuesto (Chadwick y Lowe, 1990 y 1994) se basa en: la provocación del pensamiento delirante, el análisis de la evidencia de cada creencia, la identificación del sesgo cognitivo subyacente y en el debilitamiento de dicho pensamiento delirante a través de la búsqueda de evidencia empírica en contra de las creencias. Normalmente se comienza por las ideas más periféricas del delirio y que menos resistencia provocan en el paciente, para ir avanzando de forma gradual hacia lo que constituye el núcleo de la idea delirante. Los resultados obtenidos ponen de manifiesto que estos procedimientos producen una reducción significativa en la convicción del delirio en un número importante de pacientes (Garety et al, 1994; Kingdon y Turkington, 1994) ${ }^{8}$.

\section{CASO CLÍNICO}

Benigno Martín es un sujeto de 24 años, soltero, que vive solo y trabaja como enfermero en la Clínica Privada El Bosque de Madrid.

Los datos psiquiátricos de que se disponen figuran en una única entrevista realizada por el Dr. Roncero (psiquiatra privado) a demanda del paciente.

Sus padres se divorciaron y desde los 5 años, aproximadamente, se encuentra al cuidado de su madre, que según comenta "no estaba impedida ni loca, sino que era muy perezosilla".

Estudió A.T.S y realizó por correspondencia cursos de estética, maquillaje y peluquería. Su madre ha fallecido hace 2 meses y comenta de ella que era muy guapa y que a él no le gustaba que se descuidara, por este motivo la maquillaba, la peinaba, le arreglaba las uñas, etc.

No recuerda con exactitud la fecha del divorcio de sus padres e informa que desde entonces no ha vuelto a ver a su padre, "que éste formó otra familia y que posiblemente viva en Suecia".

Nunca ha mantenido relaciones de pareja ni relaciones sexuales y considera que el único problema que tiene es la soledad.

A la exploración, consciente, eutímico, no transfiere ansiedad, pensamiento normal en curso, lenguaje fluido. Llama la atención que tras la pérdida reciente de la madre no muestre manifestaciones de duelo. Su discurso es superficial. Impresiona de cierta incapacidad de introspección.

\section{INFORMACIÓN DE OTRAS FUENTES}

Benigno ha vivido recluido en un piso añejo con una 
madre enferma. Todo lo que ha hecho en su vida ha estado encaminado a cuidar a esa mujer "perezosilla", abandonada por el esposo, posiblemente histriónica y narcisista e incapaz de dar.

Almodóvar sabiamente la omite y sólo enseña de ella una voz castrante y prohibitiva con la que impide que Benigno goce sólo, fuera de ella (j"Benigno, llevas ya media hora en la ventana"i).

Benigno no tiene amigos, posiblemente siempre ha salido de clase deprisa, como el que tiene un bebé a su cuidado y sabe que está solo, culpabilizado y entregado a un maternaje que le reorganiza y le limita la existencia.

La única conexión lúdica con el mundo es la academia de baile que hay frente a su balcón, baile que simboliza la libertad y el autodominio.

Benigno pasa su tiempo libre mirando por la ventana... "como esa mujer de la guía de Marco, frente al Malecón, esperando inútilmente, viendo cómo el tiempo pasa sin que pase nada".

Un día, el misterioso Cupido, que siempre elige a sus víctimas, lanza su flecha y Benigno se enamora de Alicia... Con esta fantasía Benigno puede soportar el tedio cotidiano: hacer las tareas domésticas, trabajar, cuidar a la madre, contenerla, consolarla... Puede soportar la reclusión de su ser. Es un amor platónico, él lo sabe, pero es su amor, su único amor.

Con el amor de Alicia viene la muerte de la madre y Benigno no hace un duelo, pero... ¿cómo se puede hacer un duelo de alguien que ya estaba muerto?, ¿cuándo murió la madre de Benigno?, ¿fue cuando la abandonó el esposo o quizá ya estaba muerta y permanecía mantenida como Lenin, gracias al esfuerzo infructuoso de Benigno?

Como todo enamorado, Benigno busca la ocasión de un encuentro con su amada, y lo consigue. Valiente y decidido se le aproxima y ésta le deja entrar en su mundo con la palabra; comparten la orfandad y Alicia le habla de sus aficiones, que desde ese momento Benigno hace suyas.

Imaginemos los pensamientos de Benigno tras ese primer encuentro: ese automatismo mental que a todo enamorado desborda, ese rebobinar hacia atrás, ese interpretar a favor, ese timos que se expande haciendo recordar la canción de Palito Ortega, cuyo estribillo rezaba "la felicidad ja ja ja ja, me la dio tu amor jo jo jo jo"... ¡Cuántos proyectos, planes, dudas, que se rechazan y se aceptan, que se mejoran!, ¡Cuántas conversaciones imaginarias con el amado!. Amar es "des-centrarse", salirse de sí-mismo y conlleva algo de locura y alineación.

Se acabó la soledad desolada e improductiva.... A Benigno se le abre un nuevo mundo con el amor. Al día siguiente de este primer encuentro, vuelve a la ventana y Alicia no está. Pasan los días y Alicia no aparece por la academia. Desesperado, llama a su casa; él sabe que su padre es psiquiatra y decide pedir una cita, necesita verla..., necesita frenar su locura.

Al terminar la entrevista psiquiátrica, en un descuido de la enfermera busca a Alicia por la casa, llegando a su habitación y cogiendo una pinza del pelo, necesita algo suyo y es, en este entrar en el mundo del otro sin permiso, cuando empieza a manifestarse Benigno como un loco, que desbordado por la "pasión" forcluye la Ley.

Benigno cree que con la posesión del otro se logra la plenitud y se vence la soledad ("sólo la interioridad del otro mitiga la soledad"), pero ignora que el otro tiene su propia mismidad, su propia vida y su propio destino y esto hace que en las relaciones con los demás el sentimiento permanente sea la incompletud. A la semana siguiente vuelve a la casa de Alicia, descorazonado, con la esperanza encogida, llama al timbre y posiblemente no contesten.

Alicia ha sufrido un accidente de tráfico y ha entrado en coma, ha sido trasladada a la clínica El Bosque y Benigno ha sido elegido como enfermero para cuidarla dada su buena reputación. La vida de Benigno ahora tiene un sentido pleno: cuidar a su amada con la esperanza de que algún día, como la bella durmiente, despierte y lo invista como su príncipe.

Benigno comienza a hacer por Alicia todo lo que no ha hecho por él; ver cine mudo, asistir a representaciones de ballet...., en definitiva, gozar.

En el espectáculo de Pina Bausch, "Café Müller", Benigno se identifica con ese hombre vestido de negro, con expresión triste, que aparta del camino de dos mujeres, con los ojos cerrados, como sonámbulas o como muertas, los posibles obstáculos a manotazos.

Benigno desea compartir su aprendizaje y las emociones que despierta en él, ese nuevo mundo que está conociendo y decide "hablar con ella", al principio como un monólogo, quizá los primeros años, monólogos llenos de esperanza...., hasta que un día su generoso inconsciente decide ponerle voz a Alicia, una voz que se va a adherir a todos los proyectos de Benigno.

Benigno reforma la casa y la amuebla con el consentimiento alucinado de su amada, y hasta borda las sábanas con las letras iniciales de ambos, quizá por deseo de ésta.

Hasta aquí las únicas consecuencias del delirio de Benigno son exquisitos cuidados materiales y humanos hacia Alicia, pero en una de las películas de cine mudo "El amante menguante" Benigno se "trastorna", quizá descubre el sexo en la pareja (sexo que estuvo ausente en su vida), se identifica con Alfredo, "gordito 
y buen tío, como él", con una madre "tremenda", quizá como la suya. Benigno proyecta en la relación de Alfredo y Amparo su relación con Alicia. En la escena de la habitación en el Hotel "Youkali" Benigno descubre que el vínculo amoroso consigue su acmé en el acto sexual ("Alfredo se queda dentro de ella para siempre", le comenta Benigno a Alicia).

El amante desea ser "el-otro" poseyéndolo. El amor es pura otreidad. El amante no solo desea estar con el otro sino fundirse con el otro.

En los sujetos normales la entrega sexual es consensuada, pero en la película del amante menguante Alfredo espera a que su amante esté dormida para entregarse a una vivencia oceánica de fusión.

Benigno, quizá interpretando en esta expresividad de Amparo que el orgasmo hace feliz a la mujer (él que siempre ha pensado "que el cerebro de las mujeres es un misterio, que a las mujeres siempre hay que tenerlas en cuenta, hablar con ellas, tener detalles, acariciarlas, recordar que existen, que están vivas, que nos importan"), sigue los pasos de Alfredo y se adentra en Alicia en pleno arrobamiento amoroso para hacerla feliz. Una violación material que no es una violación formal porque Benigno habla con ella y es correspondido por ella. Benigno quiere casarse con Alicia porque a su criterio se llevan mejor que la mayoría de los matrimonios. Niega y se niega que Alicia está en coma, en estado vegetativo, que no puede decir "sí quiero" con ninguna de las partes de su cuerpo, niega que la relación con Alicia es "un monólogo, una locura".

Condenado por violación y diagnosticado de psicópata es encarcelado. En la cárcel lee las guías de Marco y se identifica "con esa gente que no tiene nada y se lo inventa todo".

A Benigno lo único que le consuela y le da esperanza es volver a ver a su amada ("yo no puedo vivir en un mundo donde no esté Alicia, un lugar en donde no puedo tener conmigo ni su pinza del pelo", pinza con la que se recogía el pelo para bailar..., pinza que tantas veces vio asomado a la ventana).

Posteriormente, cuando toma conciencia de que salir de la cárcel es imposible y de que está condenado a renunciar a su amada, toma pastillas, dejando la siguiente carta de despedida a su único amigo: "sigue lloviendo y creo que esto es un buen presagio. Cuando Alicia tuvo el accidente también llovía. Te escribo antes de fugarme. Espero que todo lo que me he tomado sea suficiente para entrar en coma y reunirme con ella. Eres mi único amigo, te dejo la casa que preparé para Alicia y para mí. Donde sea que me lleven ven a verme y habla conmigo, no seas tan hermético, cuéntamelo todo, hasta siempre, amigo mío".

\section{JUICIO CLINICO}

Benigno es un loco de amor, de un amor delirado; es un erotómano esencial que permanece durante todo su delirio en la fase de esperanza.

\section{COMENTARIO FINAL}

En esta película Almodóvar nos muestra un delirio desbravado, dulce, femenino, sin despecho ni rencor a cuya comprensión puede acceder cualquier profano. Un delirio tan dulce como la interpretación de Caetano Veloso del "Cucurrucucú Paloma".

Benigno no se suicida, no es como esos erotómanos que ante la imposibilidad de conseguir a su amado entran en melancolía. Benigno "se fuga" de la realidad de nuevo, la ingesta de pastillas es un acto más de servidumbre al delirio.

\section{BIBLIOGRAFÍA}

1. De Portugal E, Cervilla J. Trastorno delirante. Madrid: Aula Médica S.A.; 2004.

2. Bellomo LE. La erotomanía: la expresión clínica del delirio de "ser-amado". Rev. Argent Clin Neuropsiquiatr [revista en Internet] 2005; 12(1) [consultado el 5-01-2009]. Disponible en: http// www.alcmeon.com.ar/12/45/02-_bellomo.htm.

3. Esquirol JED. "Memorias sobre las locuras y sus variedades". Madrid: Dorsa; 1991.

4. Sérieux P, Capgras J. Las locuras razonantes. El delirio de interpretación. Madrid: Ergon; 2008.

5. Kraepelin E. Introducción a la clínica psiquiátrica. Madrid: Nieva; 1988.

6. De Clérambault G. Les psychoses passionelles. Ouvre Psychiatrique. París : Press Universitaires de France ; 1921.

7. Ey H, Bernard P, Brisset CH. Tratado de psiquiatría. Barcelona: Toray-Masson S.A.; 1965.

8. Vallejo Pareja MA. Manual de terapia de conducta. Madrid: Dykinson; 1998. 